\title{
CLOSE/WIDE DEGENERACY IN CENTRAL PERTURBATIONS OF PLANETARY LENSING
}

\author{
Doeon Kim ${ }^{1}$, Cheongho Han ${ }^{1}$, And Byeong-Gon Park ${ }^{2}$ \\ ${ }^{1}$ Program of Brain Korea 21, Department of Physics, Chungbuk National University, Cheongju 361-763, \\ Republic of Korea \\ E-mail: dekim,cheongho@astroph.chungbuk.ac.kr \\ ${ }^{2}$ Korea Astronomy and Space Science Institute, Hwaam-Dong, Yuseong-Gu, Daejeon 305-348, Republic of Korea \\ E-mail: bgpark@kasi.re.kr \\ (Received April 18, 2009; Accepted June 5, 2009)
}

\begin{abstract}
We investigate the degeneracy in the pattern of central microlensing perturbations of a pair of planetary systems where the planets are located from the primary with projected separations in units of the Einstein radius $s$ and $s^{-1}$, respectively. From this, we confirm the fact that although alike, the patterns of central perturbations induced by a close $(s<1)$ planet and a wide $(s>1)$ planet are not identical and the degree of difference depends on the planet/primary mass ratio and the planet-primary separation. We find that the difference can be greater than $5 \%$ for planetary systems with lensing parameters located in the parameter space of $\left(1 / 1.8<|s|<1.8, q>5 \times 10^{-3}\right),(1 / 1.3<|s|<1.3$, $\left.q>1 \times 10^{-3}\right)$, and $\left(1 / 1.2<|s|<1.2, q>5 \times 10^{-4}\right)$, where $q$ represents the planet/primary mass ratio. Although this range occupies a small fraction of the entire parameter space of planetary systems, we predict that the chance of resolving the close/wide degeneracy would not be meager considering that the planet detection efficiency is higher for planets with resonant separations $(s \sim 1)$ and heavier masses. We also find that the differences between the perturbation patterns are basically caused by the effect of the planetary caustic. This explains the tendency of the perturbation difference where (1) the difference increases as the planet/primary mass ratio increases and the separation approaches the Einstein radius, (2) the region of major difference is confined within the region around the line connecting the central and the planetary caustics, and (3) a wide (close) planetary system has a more extended central perturbation region toward the (opposite) direction of the planet.
\end{abstract}

Key words : gravitational lensing - planets and satellites: general

\section{INTRODUCTION}

Recently, detections of eight extrasolar planets by using the microlensing method were reported (Bond et al. 2004; Udalski et al. 2005; Beaulieu et al. 2006; Gould et al. 2006; Gaudi et al. 2008; Bennett et al. 2008; Dong et al. 2008). The microlensing method is important because it is sensitive to planets that are difficult to be discovered by other methods. These planets include cool planets located beyond the snow-line (Kennedy, Kenyon, \& Bromley 2006; Bennett, Anderson, \& Gaudi 2006), low-mass planets with masses below the Earth mass (Bennet \& Rhie 1996), remote planets distributed throughout the Galaxy (Baltz \& Gondolo 2001; Chung et al. 2006), and free-floating planets unbound to host stars (Bennett \& Rhie 2002; Han et al. 2004).

The microlensing signal of a planet is a short blip or dip to the smooth and symmetric standard light curve of the primary-induced lensing event occurring on a background star (Mao \& Paczyńsky 1991; Gould \& Loeb 1992). To detect the short-duration signal,

Corresponding Author: C. Han current planetary lensing searches are being conducted by using a combination of survey and follow-up observations. Survey observations are designed to maximize the lensing event rate by monitoring a large area of sky on nightly basis and to issue alerts of lensing events in the early stage of lensing magnification by analyzing data in real time. Follow-up observations are focused on these alerted events to detect short-term planetary lensing signals. However, the number of telescopes available for follow-up observations is far less to monitor all events alerted at any given time and thus observations are concentrated on events that will maximize the probability of planet detection. Currently, the highest priority is given to highly magnified events because these events have intrinsically high planet detection efficiency due to the close passage of the source trajectory to the central perturbation region induced by the planet (Griest \& Safizadeh 1998) and followup observations can be prepared in advance due to the predictable time of the perturbation occurring near the peak of a lensing light curve. As a result, 6 out of 8 microlensing planets (OGLE-2005-BLG-071Lb, OGLE2005-BLG-169Lb, OGLE-2006-BLG-109Lb,c, MOA-20 
07-BLG-400b, MOA-2007-BLG-192) were detected through the channel of high-magnification events.

Although high-magnification events provide an efficient channel to detect planetary signals, a unique interpretation of an observed signal often becomes complicated due to possible degeneracies. There are two major degeneracies causing this complication. The first degeneracy is caused by the fact that the central perturbation of a high-magnification event can be produced not only by a planet but also by a very close or a very wide-separation binary with lens components of roughly equal masses. We refer this degeneracy as the "planet/binary" degeneracy. The other degeneracy is caused by the fact that the perturbation induced by a planet with a projected separation in units of the Einstein ring, $s$, is very similar to the perturbation induced by a planet with a separation $s^{-1}$. Hereafter, we refer the planets with separations $s<1$ and $s>1$ as a close planet and a wide planet, respectively, and the degeneracy between the signals produced by a close planet and a wide planet as the "close/wide degeneracy". Perturbations induced by a planet and a binary companion are intrinsically different and thus in most cases the planet/binary degeneracy can be resolved from detailed modelling of the signal. In some cases, the degeneracy can be resolved even without detailed modelling because a planet or a binary companion produces characteristic features in lensing light curves that cannot be mimicked by other interpretations (Han \& Gaudi 2008; Han 2009b). On the other hand, the close/wide degeneracy is known to be much more severe because the degeneracy is rooted in the similarity between the lens mapping equations of the close and the wide planetary systems (Dominik 1999; An 2005).

Although the close/wide degeneracy is severe, the patterns of the central perturbations induced by a close planet and a wide planet are not identical (Chung et al. 2005). In this paper, we investigate the degeneracy in the patterns of central perturbations in detail to find (1) the region in the parameter space where the degeneracy can be resolved, (2) the tendencies of the difference between the patterns of the perturbations induced by the two degenerate cases of planets, and (3) the cause of the difference between the patterns.

\section{PLANETARY LENSING IN GENERAL}

When a source star is gravitationally lensed by a single object, the resulting light curve of the source star flux is smooth and symmetric. However, when the star is lensed by a star with a planet, the light curve of the lensing event can have a short-duration perturbation. The perturbation occurs when the source star passes close to a caustic, that represents the source star positions at which the lensing magnification of a point source becomes infinite. The set of caustics form closed curves, each of which is composed of concave curves (fold caustics) that meet at points (cusps). For a planetary lensing case, there exist two or three sets of caustics.

The planetary caustic refers to the caustic that forms away from the primary position. The location, shape, and the number of the planetary caustics depend on the projected separation between the primary and the planet. If the separation is greater than the Einstein radius $(s>1)$, there exists a single four-cusp planetary caustic located on the primary-planet axis. If the separation is smaller than the Einstein radius $(s<1)$, on the other hand, there exist two three-cusp planetary caustics located above and below the primary-planet axis. The position vector to the center of the planetary caustic from the position of the primary, $\mathbf{r}$, is related to the position vector to the planet, $\mathbf{s}$, by $\mathbf{r}=\mathbf{s}\left(1-s^{-2}\right)$ and thus the center of the planetary caustic is located on the same and opposite sides of the planet with respect to the position of the primary for planets with $s>1$ and $s<1$, respectively. Not only the position of the planetary caustic but also the sign of the perturbation caused by the planetary caustic depends on the planetary separation (Gaudi \& Gould 1997; Wambsganss 1997; Bozza 1999; Han \& Chang 2003). If the source star passes close to the planetary caustic produced by a planet with $s>1$, the sign of the resulting planetary perturbation is positive, implying that the flux at the peak of the perturbation is larger than the adjacent smooth part of the light curve. By contrast, the sign of the perturbation is negative if source star passes close to the region between the planetary caustics produced by a planet with $s<1$.

The central caustic refers to the small caustic located near the position of the primary lens. The central caustic has four cusps. The three of the four cusps lean toward the primary direction and thus the central caustic has a wedge-like shape. Unlike the dramatic differences in the location, the shape, and the number of planetary caustics between the planetary systems with separations $s$ and $s^{-1}$, the central caustics produced by a pair of a close planet and a wide planet are very similar. As a result, the pattern of the central perturbations are also alike.

\section{CENTRAL PERTURBATIONS OF CLOSE AND WIDE PLANETS}

Although similar, the central caustics of the close and the wide planets and the resulting pattern of central perturbations are not exactly same. To see how the patterns of the perturbations are different, we produce maps of magnification excess, which is defined by,

$$
\epsilon=\frac{A-A_{0}}{A_{0}}
$$

where $A$ and $A_{0}$ represent the lensing magnifications with and without the planet, respectively.

In addition to the basic perturbation pattern, another factor that affects the pattern is the finite-source effect. The finite-source effect is especially important 

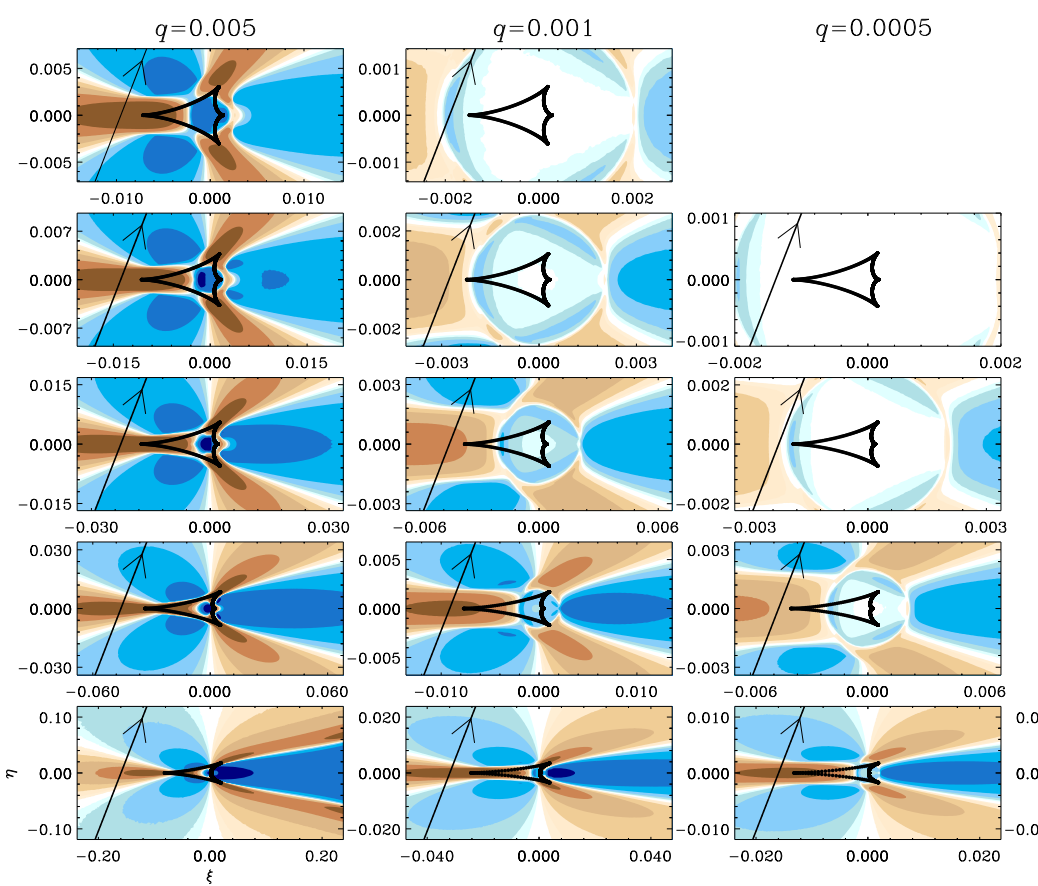

$q=0.0001$

$s=1 / 2.0$
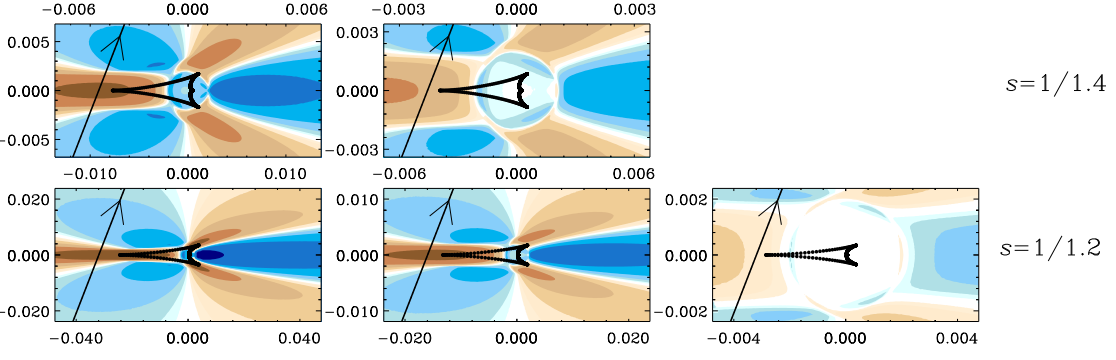

Fig. 1. - Color-scale maps of magnification excess for planets with projected separations from the primary star less than the Einstein radius. Each map is centered at the position of the photo-center and the planet is located on the left side. All lengths are scaled by the Einstein radius. Colors of the scale are chosen such that the regions with brown and blue-tone colors represent the areas where the magnification is higher and lower than the single-lensing magnification without the planet, respectively. For each tone, the color scale becomes darker at the excess levels of $|\epsilon|=1 \%, 2 \%, 4 \%, 8 \%, 16 \%$, and $32 \%$, respectively. Maps are presented only for planetary systems detectable through the channel of high-magnification events. The labels above and on the right side represent the planet/primary mass ratios and the planet-primary separations, respectively.

for central perturbations because the source size of a typical Galactic lensing event is not negligible compared to the size of the central caustic. The lensing magnification affected by the finite-source effect corresponds to the magnification averaged over the source star surface. Due to the finite-source effect, the perturbation pattern is smoothed out (Bennett \& Rhie 1996).

For the construction of the perturbation-pattern maps including the finite-source effect, we use a rayshooting method. In this method, a large number of rays are uniformly shot from the image plane through the lens plane and then collected in the source plane. Then, the magnification is obtained by the ratio of the number density of shots on the source plane to that on the image plane (Schneider \& Weiss 1986; Kayser, Refsdal, \& Stabell 1986; Wambsganss 1997). On the basis of this method, we improve the routine by adopting an algorithm developed by (Dong et al. 2006), in which two major factors contribute to the acceleration of the computation. The first factor is that the algorithm limits the range of ray shooting on the image plane to a narrow annulus encircling the Einstein ring of the primary lens. This is based on the fact that only rays within the annulus arrive at the central perturbation region on the source plane. The second factor is that the algorithm keeps the information of the positions of all the rays arriving at the target region in the buffer memory space so that the information can be promptly used to compute the magnification of a finite source, which is computed by counting the number of rays arriving within the boundary of the source star. In the computation, we consider the limb darkening of the source star by modelling the source brightness profile as

$$
\frac{I}{\langle I\rangle}=1-\Gamma\left(1-\frac{3}{2} \cos \theta\right)-\lambda\left(1-\frac{5}{4} \cos ^{1 / 2} \theta\right)
$$

where $\langle I\rangle$ is the mean surface brightness and $\theta$ is the angle between the normal direction to the source-star surface and the line of sight. We adopt a source radius normalized by the Einstein radius of $\rho_{*}=0.002$ and a linear and a square-root limb-darkening coefficients of $(\Gamma, \Lambda)=(-0.46,1.11)^{*}$ by taking these of a typical bright main-sequence source star.

In Figure 1 and 2, we present the constructed excess maps for various planetary systems, where the maps presented in Figure 1 are for close planetary systems while the maps in Figure 2 are for wide planetary systems. Each map is centered at the position of the photo-center, which located at the position with a po-

\footnotetext{
* from Claret's limb darkening coefficients table in Astronomica databases Vizier (http://vizer.cfa.harvard.edu/viz-bin/VizieR)
} 


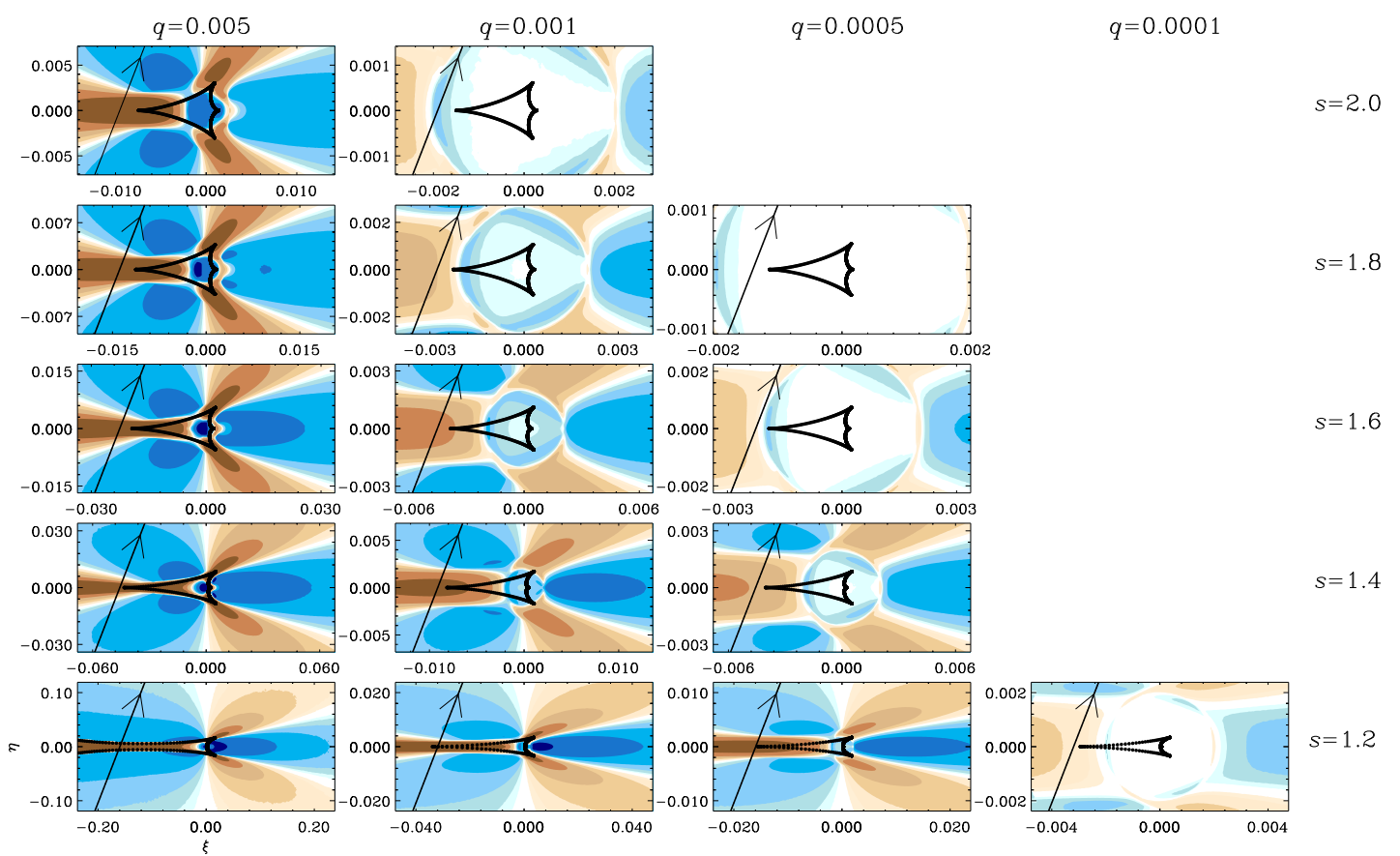

Fig. 2.- Color-scale maps of magnification excess for planets with projected separations from the primary star greater than the Einstein radius. Notations are same as in Fig. 1.

sition vector from the location of the primary lens of

$$
\delta \boldsymbol{\xi}= \begin{cases}\mathbf{s}^{-1} q /(1+q) & \text { for } s>1 \\ -\mathbf{s}\left[(1+q)^{-1}-1\right] & \text { for } s<1\end{cases}
$$

The abscissa of the map is parallel with the planetprimary axis and the planet is located on the left side. All lengths the normalized by the Einstein radius of the primary. Colors of the scale are chosen such that the regions with brown and blue-tone colors represent the areas where the magnification is higher and lower than the single-lensing magnification without the planet, respectively. For each tone, the color scale becomes darker at the excess levels of $|\epsilon|=1 \%, 2 \%, 4 \%, 8 \%$, $16 \%$, and $32 \%$, respectively. We present maps only for planets that can be detectable through the channel of high-magnification events by adopting the criteria of Han (2009a), i.e., the central caustic should be larger than $1 / 4$ of the source size.

From the comparison of the maps, it is found that, as expected, the perturbation patterns of each pair of a close and a wide planetary systems are alike. However, it is also found that slight differences exist between the maps. To better see the difference in the magnification pattern, we construct additional maps showing the pattern of the difference between magnification excesses of the close and wide planetary systems, i.e.,

$$
\Delta \epsilon=\epsilon_{C}-\epsilon_{W},
$$

where $\epsilon_{C}$ and $\epsilon_{W}$ are excesses for planetary systems with a close planet and a wide planet, respectively. The maps of excess difference are presented in Figure 3. In Figure 4, we also present the curves of excess difference resulting from the source trajectories marked in the corresponding panels in Figure $1-3$.

From the comparison of the excess patterns, we find the following tendencies. First, we confirm that although similar, the patterns of perturbations induced by a close planet and a wide planet are not identical and the magnification difference becomes larger as the planet/primary mass ratio increases and the primary-planet separation approaches the Einstein radius (Chung et al. 2005). We find that the difference can be greater than $5 \%$ for planetary systems with lensing parameters located in the parameter space of $\left(1 / 1.8<|s|<1.8, q>5 \times 10^{-3}\right),(1 / 1.3<|s|<1.3$, $\left.q>1 \times 10^{-3}\right)$, and $\left(1 / 1.2<|s|<1.2, q>5 \times 10^{-4}\right)$. This range occupies a small fraction of the entire parameter space of planetary systems. However, we note that considering high planet-detection efficiencies for resonant $(s \sim 1)$ and heavier planets, it is expected that the chance of resolving the close/wide degeneracy would not be meager.

Second, for a given pair of a close planet and a wide planet, it is found that the region of major difference is confined in the region around the line connecting the central and planetary caustics. Within this region, the difference is such that the perturbation extends toward the direction of the planetary caustic. As a result, a wide planetary system has more extended perturbation region toward the direction of the planet from the position of the central caustic, while a close planetary system has more extended perturbation region toward the opposite direction of the planet.

The tendencies of the perturbation patterns of close and wide planets strongly suggest that the difference 

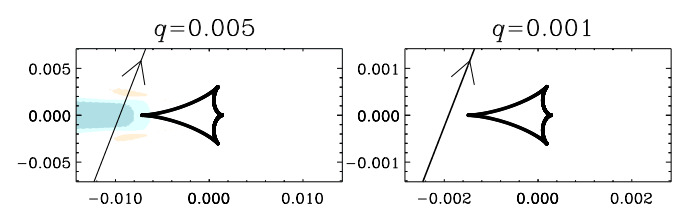

$q=0.0005$

$q=0.0001$
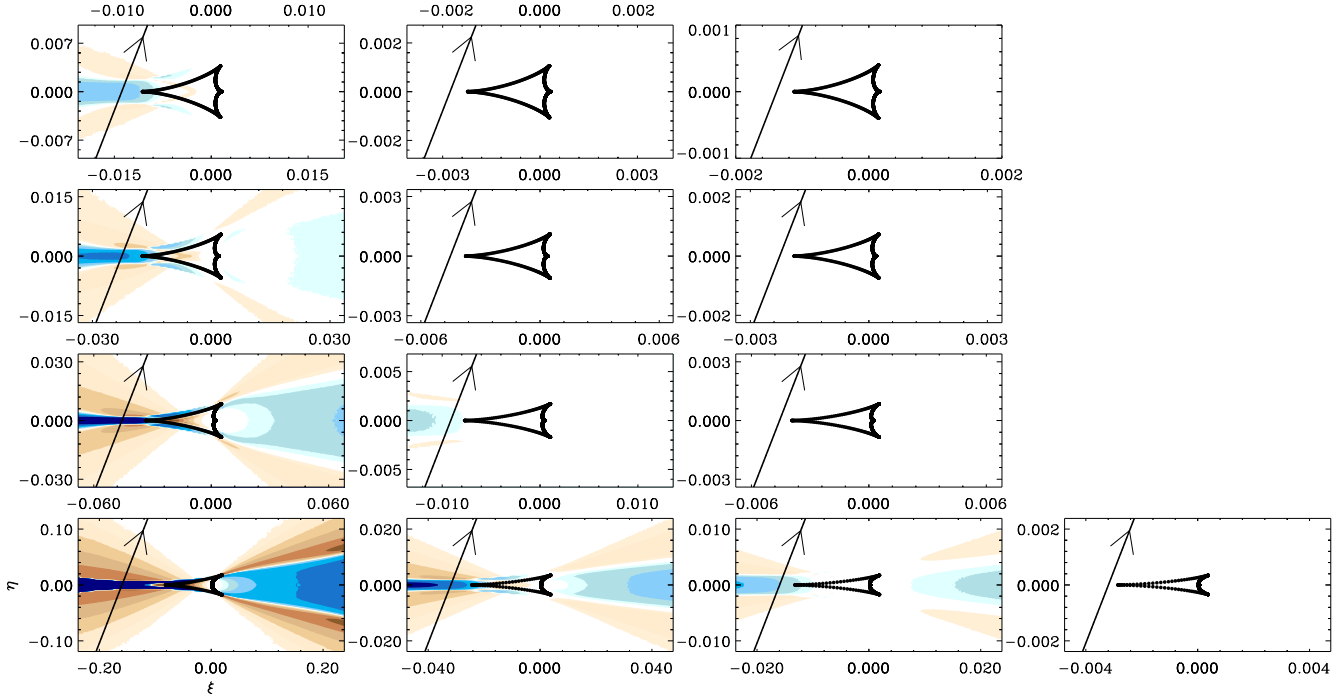

$s=1.2,1 / 1.2$

Fig. 3.- Color-scale maps of the difference in the magnification excess between close and wide planetary systems. Regions with brown and blue-tone colors represent the areas where the magnification of a close planetary system is greater and less than the magnification of a wide planetary system, respectively. The straight line with an arrow in each panel represents the source trajectory where the resulting curve of excess difference is presented in the corresponding panels in Fig. 4.

is caused by the effect of the planetary caustic. To better see the effect of the planetary caustic on the central perturbation, we plot an additional maps of excess and excess difference in a range including the perturbation regions induced by the planetary caustics. The maps are presented in Figure 5. From the the figure, it is found that the region of central perturbation is not isolated and instead it is connected to the region of perturbation induced by the planetary caustic, implying that the perturbation induced by the planetary caustic has effect on the central perturbation. As the planet/primary mass ratio increases and the planetprimary separation approaches the Einstein radius, the planetary caustic becomes bigger and thus its effect on the central perturbation region increases. This matches the tendency of the difference between the central perturbations induced by a close planet and a wide planet.

\section{CONCLUSION}

We investigated the close/wide degeneracy in the patterns of central planetary perturbations. From this, we confirmed that although alike, the perturbation patterns induced by a pair of a close planet and a wide planet are not exactly same and the degree of difference depends on the planet/primary mass ratio and the planet-primary separation. We found that the difference can be greater than $5 \%$ for planetary systems with lensing parameters located in the parameter space of $\left(1 / 1.8<|s|<1.8, q>5 \times 10^{-3}\right),(1 / 1.3<|s|<1.3$, $\left.q>1 \times 10^{-3}\right)$, and $\left(1 / 1.2<|s|<1.2, q>5 \times 10^{-4}\right)$. Although this range occupies a small fraction of the en- tire parameter space of planetary systems, we predict that the chance of resolving the close/wide degeneracy would not be meager considering that the planet detection efficiency is higher for resonant and heavier planets. We also found that the differences between the perturbation patterns are basically caused by the effect of the planetary caustic. This explains the tendency of the perturbation difference where (1) the difference increases as the planet/primary mass ratio increases and the separation approaches the Einstein radius, (2) the region of major difference is confined within the region around the line connecting the central and planetary caustics, and (3) a wide (close) planetary system has a more extended central perturbation region toward the (opposite) direction of the planet.

\section{ACKNOWLEDGEMENTS}

This work was supported by the research grant of the Chungbuk National University in 2008.

\section{REFERENCES}

An, J. H., 2005, Gravitational lens under perturbations: symmetry of perturbing potentials with invariant caustics, MNRAS, 356, 1409

Baltz, E. \& Gondolo, P., 2001, Binary Events and Extragalactic Planets in Pixel Microlensing, ApJ, 559, 41

Beaulieu, J. P., et al., 2006, Discovery of a cool planet of 5.5 Earth masses through gravitational microlensing, Nature, 439, 437 


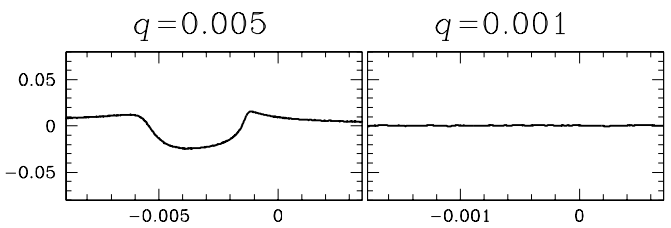

$$
q=0.0005
$$

$q=0.0001$
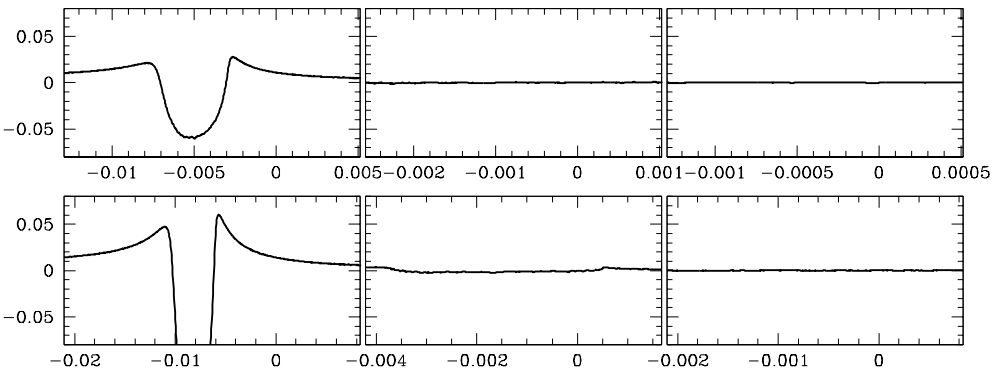

$S=1.6,1 / 1.6$

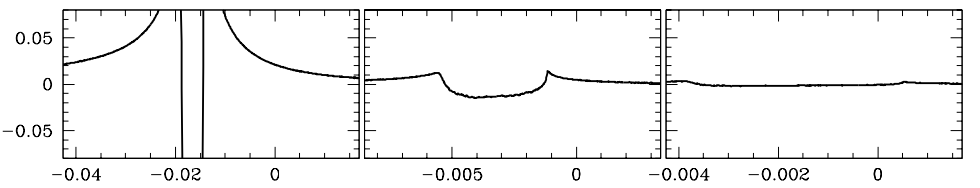

$S=1.4,1 / 1.4$

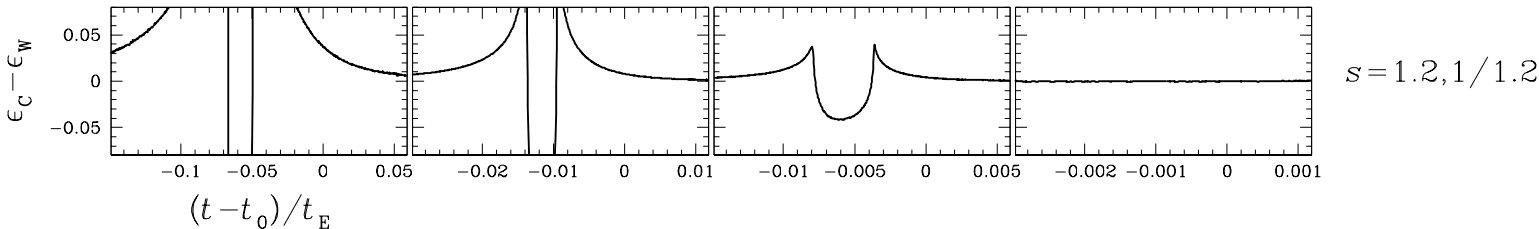

Fig. 4. - Curves of excess difference between the light curves produced by pairs of close and wide planetary systems. The source trajectory of the event responsible for each curve is presented in the corresponding panels in Figure $1-3$.

Bennett, D. P., Anderson, J., \& Gaudi, B. S., 2006, Characterization of Gravitational Microlensing Planetary Host Stars, AAS, 209, 152.04

Bennett, D. P. \& Rhie, S. H., 1996, Detecting EarthMass Planets with Gravitational Microlensing, ApJ, 472,660

Bennett, D. P. \& Rhie, S. H., 2002, Simulation of a Space-based Microlensing Survey for Terrestrial Extrasolar Planets, ApJ, 574, 985

Bennett, D. P., et al. 2008, Simulation of a Space-based Microlensing Survey for Terrestrial Extrasolar Planets, ApJ, 684, 663

Bond, I. A., et al., 2004, OGLE 2003-BLG-235/MOA 2003-BLG-53: A Planetary Microlensing Event, ApJ, 606, L155

Bozza, V., 1999, Perturbative analysis in planetary gravitational lensing, A\&A, 348, 311

Chung, et al., 2005, Properties of Central Caustics in Planetary Microlensing, ApJ, 630, 535

Chung, et al., 2006, The Possibility of Detecting Planets in the Andromeda Galaxy, ApJ, 650, 432

Dominik, M., 1999, The binary gravitational lens and its extreme cases, A\&A, 349, 108

Dong, S., et al., 2006, Planetary Detection Efficiency of the Magnification 3000 Microlensing Event OGLE2004, ApJ, 642, 842
Dong, S., et al., 2009, Microlensing Event MOA-2007BLG-400: Exhuming the Buried Signature of a Cool, Jovian-Mass Planet, ApJ, 698, 1826

Gaudi, B. S., \& Gould, A. 1997, Planet Parameters in Microlensing Events, ApJ, 486, 85

Gaudi, B. S., et al., 2008, Discovery of a Jupiter/Saturn Analog with Gravitational Microlensing, Science, 319,927

Gould, A. \& Loeb, A., 1992, Discovering planetary systems through gravitational microlenses, ApJ, 396, 104

Gould, A., et al., 2006, Microlens OGLE-2005-BLG-169 Implies That Cool Neptune-like Planets Are Common, ApJ, 644, L37

Griest, K. \& Safizadeh, N., 1998, The Use of HighMagnification Microlensing Events in Discovering Extrasolar Planets, ApJ, 500, 37

Han, C., 2009a, Distinguishing Between Planetary and Binary Interpretations of Microlensing Central Perturbations Under the Severe Finite-Source Effect, ApJ, 691, 9

Han, C., 2009b, Microlensing Zone of Planets Detectable Through the Channel of High-Magnification Events, ApJ, 691, 452

Han, C. \& Chang, K., 2003, Signs of Planetary Microlensing Signals, ApJ, 597, 1070 


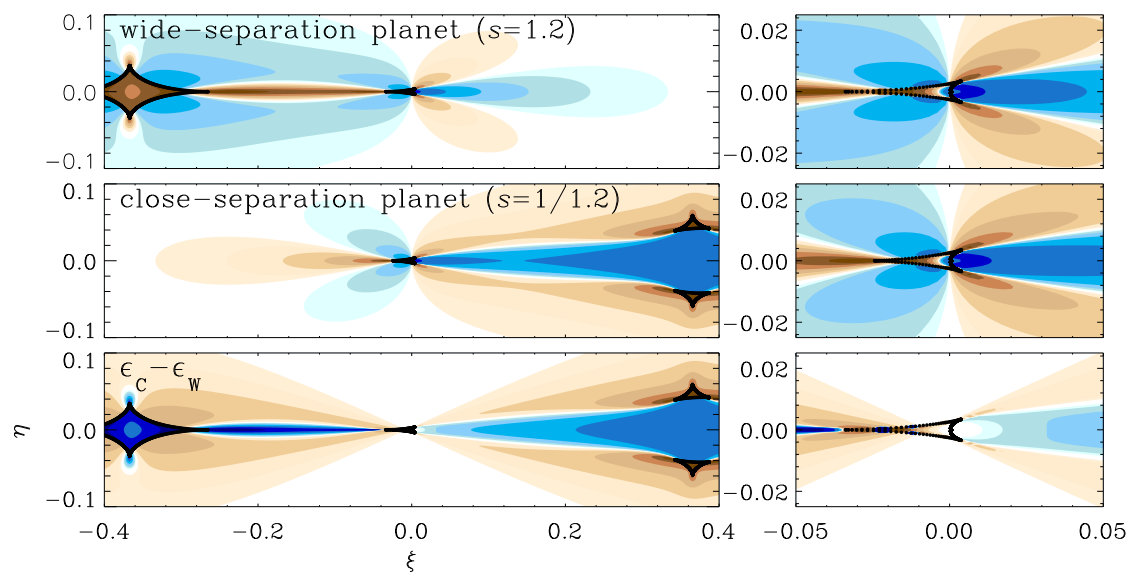

Fig. 5. - Maps of magnification excess (upper and middle panels) and excess difference (bottom panel) for an example pair of a close planet and a wide planet. The left panels show the pattern in the range including the perturbation regions induced by the planetary caustics. The right panels show the blowups of the central perturbation region. The planet/primary mass ratio is $q=0.001$ and the projected planet-primary separations are $s=1 / 1.2$ and 1.2 for the close and wide planetary systems, respectively. To show the basic perturbation pattern, maps are constructed assuming a point source.

Han, C. \& Gaudi, B. S., 2008, A Characteristic Planetary Feature in Double-Peaked, High-Magnification Microlensing Events, ApJ, 689, 53

Han, C., et al., 2004, Gravitational Microlensing: A Tool for Detecting and Characterizing Free-Floating Planets, ApJ, 604, 372

Kayser, R., Refsdal, S., \& Stabell, R., 1986, Astrophysical applications of gravitational micro-lensing, A\&A, 166,36

Kennedy, G. M., Kenyon, S. J., \& Bromley, B. C., 2006, Planet Formation around Low-Mass Stars: The Moving Snow Line and Super-Earths, ApJ, 650, L139

Mao, S. \& Paczyński, B., 1991, Gravitational microlensing by double stars and planetary systems, ApJ, 374, L37

Schneider, P. \& Weiss, A., 1986, The two-point-mass lens - Detailed investigation of a special asymmetric gravitational lens, A\&A, 164, 237

Udalski, A., et al., 2005, A Jovian-Mass Planet in Microlensing Event OGLE-2005-BLG-071, ApJ, 628, L109

Wambsganss, J., 1997, Discovering Galactic planets by gravitational microlensing: magnification patterns and light curves, MNRAS, 284, 172 